\title{
Res-Parity: Parity Violation in Inelastic scattering at Low $Q^{2}$
}

\author{
Paul E. Reimer ${ }^{1}$, P.E. Bosted ${ }^{2}$, J. Arrington ${ }^{1}$, H. Mkrtchyan ${ }^{3}$, and X. Zheng ${ }^{4}$ \\ 1 Physics Division, Argonne National Laboratory, Argonne, IL 60439 USA \\ 2 Thomas Jefferson National Accelerator Facility, Newport News, VA 23606, USA \\ 3 Yerevan Physics Institute, Yerevan, Armenia \\ 4 Massachusetts Institute of Technology, Cambridge, MA 02139, USA
}

Received: September 21, 2006

\begin{abstract}
Parity violating electron scattering has become a well established tool which has been used, for example, to probe the Standard Model and the strange-quark contribution to the nucleon. While much of this work has focused on elastic scattering, the RES-Parity experiment, which has been proposed to take place at Jefferson Laboratory, would focus on inelastic scattering in the low- $Q^{2}$, low- $W$ domain. RES-Parity would search for evidence of quark-hadron duality and resonance structure with parity violation in the resonance region. In terms of parity violation, this region is essentially unexplored, but the interpretation of other high-precision electron scattering experiments will rely on a reasonable understanding of scattering at lower energy and low- $W$ through the effects of radiative corrections. RES-Parity would also study nuclear effects with the weak current. Because of the intrinsic broad band energy spectrum of neutrino beams, neutrino experiments are necessarily dependent on an untested, implicit assumption that these effects are identical to electromagnetic nuclear effects. RES-Parity is a relatively straight forward experiment. With a large expected asymmetry $\left(\approx 0.5 \times 10^{-4}\right)$ these studies may be completed with in a relatively brief period.
\end{abstract}

PACS. 13.60-r Photon and charged-lepton interactions with hadrons - 11.30.Er Charge conjugation, parity, time reversal, and other discrete symmetries $-24.30 .-\mathrm{v}$ Resonance reactions $-12.15 . \mathrm{Mm}$ Neutral Currents - 12.15.-y Electroweak interactions

\section{Introduction}

Until recently, the electromagnetic interaction has been the fundamental probe of the structure of the nucleon. It has been used in many kinematic domains from elastic scattering through deep inelastic scattering (DIS). In between these two extremes lies the nucleon resonance region. This region has also been well studied with the electromagnetic interaction with many interesting results, including quark-hadron duality in which the structure of the nucleon resonances remarkably averages to be the same as the DIS structure, indicating a cancellation of higher twist terms [1].

The weak interaction has also been used with high energies with neutrino beams to study the quark structure of the proton in DIS kinematics, but with reasonable precision possible only on nuclear targets. With recent advances in polarized electron parity violating experiments, it has become possible to use the weak force as a probe of nucleon structure in other kinematic regimes as well. Several experiments, most recently the HAPPEX [2] and G0 [3] experiments at Thomas Jefferson National Accelerator Facility (JLab), have used this technique to explore the effects of intrinsic strangeness in the proton through parity violation in elastic scattering.

Building on the recent success of experiments measuring parity violation at JLab and elsewhere, the ResParity experiment [4] has been proposed to explore parity violation in the resonance region $\left(Q^{2}<1 \mathrm{GeV}^{2}\right.$ and $\left.M_{\text {proton }}^{2}<W^{2}<4 \mathrm{GeV}^{2}\right)$. These studies would not only examine the nucleon's parity violating response in the resonance region and study quark-hadron duality, but also study the nuclear dependence of parity violation with deuterium and carbon targets.

\section{Parity Violation and the Resonance Region}

Lepton-nucleon scattering takes place either through the exchange of a photon or a $Z^{0}$. At energies well below the mass of the $Z^{0}$ boson, the electromagnetic interaction is dominant and the $Z^{0}$-exchange and interference terms can easily be neglected in the unpolarized cross section. The electromagnetic part is parity conserving, however, so that in a measurement of parity violating effects, the normally dominate electromagnetic term would not contribute to the measured asymmetry. 
This asymmetry was first measured in the DIS region by Prescott et al. at SLAC [5,6] and served the establish the Standard Model of the electroweak interaction. In the DIS region, the asymmetry can be expressed in terms of the Standard Model couplings $C_{1 q}$ (axial electron $\times$ vector quark) and $C_{2 q}$ (vector electron $\times$ axial quark) which depend on $\sin ^{2} \theta_{W}$ and the quark distribution functions of the target $[7,8]$. Ignoring the strange and charmed quark contribution, on an isoscalar target, such as deuterium or carbon, this asymmetry is

$A_{\mathrm{DIS}}^{D}=-\left(\frac{3 G_{F} Q^{2}}{2 \sqrt{2} \pi \alpha}\right) \frac{\left(2 C_{1 u}-C_{1 d}\right)+Y\left(2 C_{2 u}-C_{2 d}\right) R_{v}}{5+R_{v}}$,

where $Y$ is a kinematical factor, and $R_{v}$ is the ratio of valence up and down quark distributions to the total up and down quark and antiquark distributions. Assuming that quark-hadron duality (see Sec. 2.1) holds, this would give an asymmetry of $A^{H} / Q^{2} \approx 50 \times 10^{-6}$ for hydrogen and $A^{D, C} / Q^{2} \approx 65 \times 10^{-6}$ for deuterium and carbon.

The asymmetry in the resonance region, $A_{\text {Res }}$, can be described in terms of response functions to specific final states, $R(q, \omega)$, combined with a non-resonant background as $[9-12]$

$$
A_{\mathrm{Res}}=A_{0} Q^{2} \frac{v_{L} R_{L}^{A V}(q, \omega)+v_{T} R_{T}^{A V}(q, \omega) v_{T^{\prime}} R_{T^{\prime}}^{V A}(q, \omega)}{v_{L} R_{L}(q, \omega)+v_{T} R_{T}(q, \omega)},
$$

with $A_{0} \approx 6.5 \times 10^{-4}$. Here $v$ is a lepton kinematic factor and the subscripts $L, T$ and $T^{\prime}$ denote longitudinal, transverse and axial, respectively. The superscript $A V(V A)$ represent the interference between axial-vector (vector) leptonic currents and the vector (axial-vector) hadronic currents. This general formalism has only been worked out in detail for elastic scattering [7] and for the $\mathrm{N} \rightarrow \Delta(1232)$ transition $[7,13,14]$

A recent calculation of parity violating electron scattering in the $\mathrm{N} \rightarrow \Delta$ transition has been made Matsui, Sato and Lee [15]. These predictions are based on a dynamical model of pion electroweak pion production reactions [16]. In this framework, the asymmetry may be expressed as

$$
\begin{aligned}
A_{\mathrm{Res}}^{\Delta(1232)} & =-\frac{Q^{2} G_{F}}{4 \sqrt{2} \pi \alpha}\left(2-4 \sin ^{2} \theta_{W}+\Delta_{V}+\Delta_{A}\right) \\
& \approx-Q^{2}\left(8.99 \times 10^{-5}\right)\left(1.075+\Delta_{V}+\Delta_{A}\right),
\end{aligned}
$$

where $Q^{2}$ is in units of $\mathrm{GeV}^{2}$. Here, $\Delta_{A}$ depends on the $W_{3}$ structure function with contributions only from the axial vector part of the neutral current and $\Delta_{V}$ depends on the $W_{1,2}$ structure functions with contributions from the vector part of the neutral current. Near the $\Delta(1232)$ resonance, the $\Delta_{A}$ term is dominant over the $\Delta_{V}$ term. The non-resonant contribution to the $\Delta_{A}$ term is minimal. This allows for the extraction of the axial form factors of the $\mathrm{N} \rightarrow \Delta$ transition from $A_{\text {Res }}$.

In studying the resonance region, Res-Parity would explore quark-hadron duality and the EMC effect with parity violation. It is important to remember that essentially no data exists to guide predictions, and so it is quite probable that significant surprises exist.

\subsection{Duality and Higher Twist}

Electron scattering experiments have shown that the unpolarized structure functions, when measured in the resonance region give on average the same result when measured at significantly higher $Q^{2}$ in DIS kinematics if the proper scaling variable is chosen [17]. This phenomena is known as quark-hadron duality and has also been shown to hold in separated longitudinal and transverse structure function and spin structure functions [1]. Quark-hadron duality can be described in terms of an operator product expansion (OPE) $[18,1]$ of the moments of the structure functions. The leading twist terms in this expansion represent scattering from individual quarks in the target, while higher twist terms take into account quark-gluon correlations. In order for duality to hold, these higher twist terms must either cancel or be negligible. The model of Matsui, Sato and Lee, discussed above, predicts a $40 \%$ or greater departure from duality for the this parity violating asymmetry, while predicting duality for the unpolarized structure functions, in agreement with data for the $\mathrm{N} \rightarrow \Delta$ transition. This prediction is show in Fig. 1 as a function of the Nachman scaling variable,

$$
\xi=\frac{2 x}{1+\sqrt{1+4 x^{2} m_{N}^{2} / Q^{2}}},
$$

where $x$ is the Bjorken scaling variable and $m_{N}$ is the nucleon mass.

\subsection{Nuclear Ratios and the EMC Effect}

The nuclear EMC effect in the resonance region would also be studied in this experiment through parity-violation. In addition to hydrogen, the experiment plans to collect data with deuterium and carbon targets. The parity violating asymmetry has very different sensitivity to the underlying quark structure than the traditional electromagnetic probes that have been used to study the EMC effect in the past. Electron scattering is proportional to the parton distributions weighted by their charge squared, thus emphasizing the effects of the $u$-quarks and antiquarks over the $d$-quarks and antiquarks. The weak probe has a different weighting. If the EMC effect is the result of identical modifications of both quark valence and sea and antiquark distributions, then there would be an identical effect for both the photon and $Z^{0}$ exchange, yielding no observable effect in the ratios of the parity violating asymmetries. Any observed effect would then necessarily point to something beyond simple rescaling of the quark distributions, for example a flavor dependence, additional nuclear dependencies related to $Z^{0}$ exchange, or a difference in the valence-sea dependence. With respect to the latter, it is interesting to note that the Fermilab E772 Drell-Yan experiment, which is sensitive to the sea quark distributions, searched for but did not observe any large nuclear effects aside from shadowing in the sea quark distributions [19]. 


\section{Impact on other Measurements}

The measurement proposed by the Res-Parity experiment would also be important to other experiments in controlling their systematic uncertainties. Three such experiments at JLab are DIS parity violation family of experiments: the $6 \mathrm{GeV}$ PV-DIS [20,21], the $12 \mathrm{GeV}$ DISParity [22] and the $12 \mathrm{GeV}$ Large Solid Angle Spectrometer program under discussion for Hall A [23]. One of the largest uncertainties in interpreting the measured asymmetries from these experiments is likely to be from the effects of higher twist contributions. By measuring parity violation in the resonance region and testing duality in parity violation, Res-Parity would be able to limit or measure these contributions. Because of beam energy and rate limitations, all of these experiments would have data very near the resonance region. Radiative corrections to the DIS measurements would depend on input from these proposed measurements of parity violation in the resonance region by the Res-Parity experiment.

The proposed measurement would also aid in understanding the backgrounds the recently completed SLAC E158 Møller experiment [24]. This experiment is one of the most precise tests of the Standard Model below the $Z$ pole. The inelastic electron-proton scattering background was both the largest correction to the Møller asymmetry and had the largest systematic uncertainty. Direct measurements by Res-Parity would help understand this correction and reduce the systematic uncertainty assigned to it. This would also be extremely important for any future Møller scattering parity violation experiments at, for example, the $12 \mathrm{GeV}$ JLab facility $[25,26]$.

Because of the small weak cross sections, neutrino scattering experiments have, for the most part, depended on heavy nuclear target to achieve luminosities sufficient for their measurements. The weak-interaction nuclear effects in these targets is at best poorly understood and generally treated as identical to the electromagnetic nuclear effects. The Res-Parity experiment would directly measure the nuclear effects with the weak interaction. While the data would be in the resonance region rather than in DIS kinematics, this is precisely the data which the current generation of neutrino scattering experiment needs. The kinematics of these experiments is dictated by the neutrino oscillation parameters given by nature. This, and the broadband nature of most neutrino beams puts much of their kinematical coverage within the resonance region.

\section{Res-Parity Implementation in Jefferson Lab Hall A}

The Res-Parity experiment would take advantage of the significant work which has gone into previous parity violation experiments at JLab. The experiment would use essentially the same experimental configuration and fast data acquisition as the $6 \mathrm{GeV}$ PV-DIS E04-012 experiment $[20,21]$. The experiment is proposed to take place
Table 1. This table lists the proposed kinematics for the measurement on each target as well as the expected counting rate (based on a DIS calculation) the asymmetry and the statistical uncertainty which would be achieved by the proposed experiment.

\begin{tabular}{cccccc}
\hline $\begin{array}{c}W \\
(\mathrm{GeV})\end{array}$ & $x$ & $\begin{array}{c}Q^{2} \\
\left(\mathrm{GeV}^{2}\right)\end{array}$ & $\begin{array}{c}\text { rate } \\
(\mathrm{MHz})\end{array}$ & $\begin{array}{c}A \\
(\mathrm{ppm})\end{array}$ & $\begin{array}{c}d A / A \\
(\text { stat \%) }\end{array}$ \\
\hline \multicolumn{6}{c}{ hydrogen } \\
\hline 2.00 & 0.17 & 0.64 & 0.5 & 46 & 7.0 \\
1.78 & 0.24 & 0.73 & 0.5 & 51 & 6.5 \\
1.52 & 0.36 & 0.82 & 0.6 & 53 & 4.3 \\
1.21 & 0.61 & 0.91 & 0.8 & 52 & 4.2 \\
\hline \multicolumn{6}{c}{ deuterium } \\
\hline 2.00 & 0.17 & 0.64 & 0.8 & 61 & 4.5 \\
1.78 & 0.24 & 0.73 & 0.9 & 64 & 3.6 \\
1.52 & 0.36 & 0.82 & 0.9 & 67 & 3.1 \\
1.21 & 0.61 & 0.91 & 1.3 & 71 & 2.4 \\
\hline \multicolumn{6}{c}{ carbon } \\
\hline 2.00 & 0.17 & 0.64 & 0.5 & 61 & 4.6 \\
1.78 & 0.24 & 0.73 & 0.5 & 64 & 3.8 \\
1.52 & 0.36 & 0.82 & 0.5 & 67 & 3.4 \\
1.21 & 0.61 & 0.91 & 0.9 & 71 & 2.5 \\
\hline \hline
\end{tabular}

in JLab Hall A using both HRS spectrometers [27] positioned at $12.5^{\circ}$ (the smallest angle available without septum magnets) with a $85 \mu \mathrm{A} 4.8 \mathrm{GeV}$ electron beam polarized at $80 \%$ incident on liquid hydrogen, liquid deuterium and carbon targets. The incident beam energy and spectrometer angle were chosen to optimize the coverage of the resonance region without the need for additional electron beam energies or spectrometer angle changes. For each target, the measured kinematics as well as the expected statistical uncertainties are shown in Tab. 1. The expected results and statistical uncertainties are plotted in Fig. 1. To obtain this kinematic coverage, the central momenta of the spectrometers would be varied over a range of 3.0 to $4.0 \mathrm{GeV}$. To obtain the desired statistical uncertainties and to allow for studies of systematic effects, 30 days of beam time ( 9 days for hydrogen, 8 for deuterium, 12 for carbon and 1 day for configuration changes and systematic effect studies) are required.

Depending on the kinematic bin, the pion to electron ratio may be up to $0.6: 1$ (based on pion photoproduction data [28]) so contamination could represent a significant background. Pions would be identified by using a $\mathrm{CO}_{2}$ gas threshold Cherenkov detector and double-layered lead-glass shower counters in each HRS spectrometer. The combination of these two devices should provide a pion rejection of better than $10^{-4}$ with an electron identification efficiency of better than $99 \%$. In addition, both the FADC DAQ and scaler DAQ described below would measure the pion asymmetry with precision roughly equal to the electron asymmetry so that corrections may be applied if necessary.

The need to separate electron signals from pionic background forces this to be a counting experiment. Event rates of up to $1.3 \mathrm{MHz}$ are expected in this experiment. Two methods are being explored to handle these rates. 


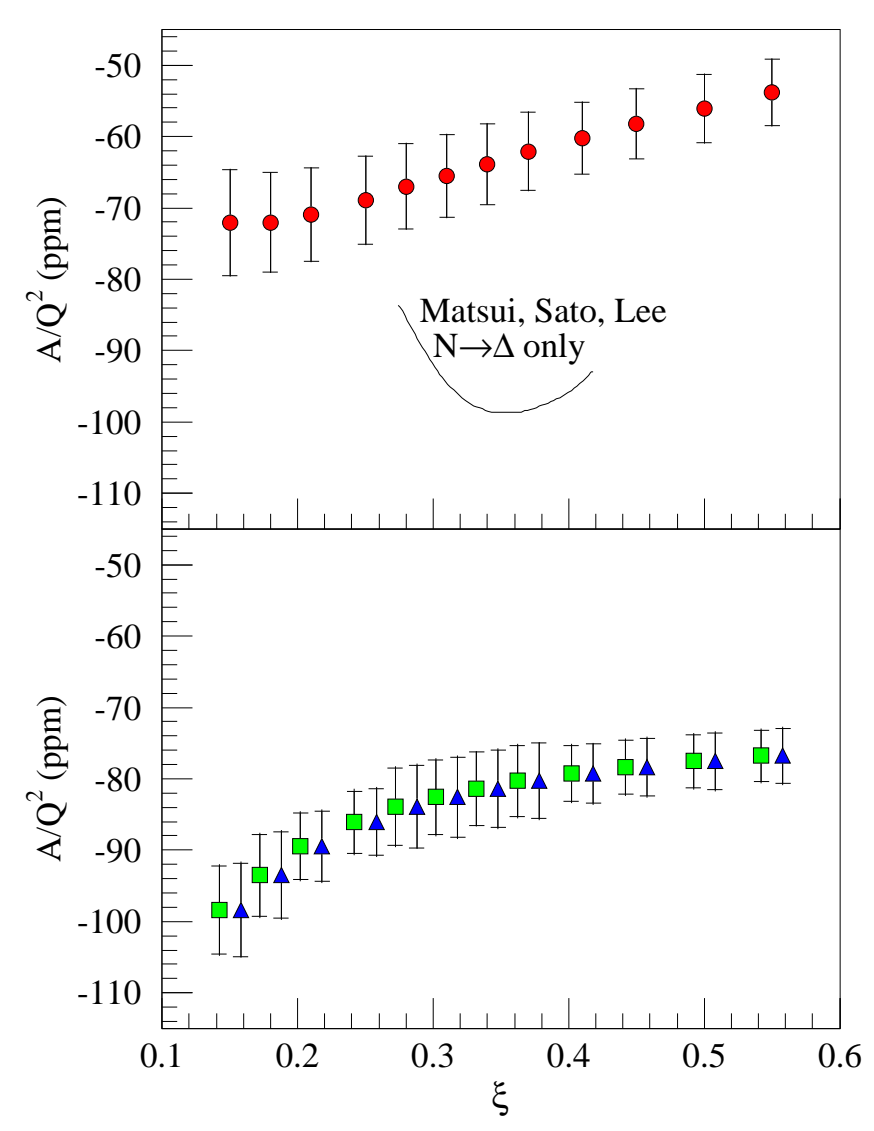

Fig. 1. The expected statistical uncertainties are shown in this figure for hydrogen (circles-top plot), deuterium (squaresbottom plot) and carbon (triangles-bottom plot) as a function of the Nachman scaling variable $\xi$. The points are plotted along the prediction of a simplified (purely $I=1$ transition) model of the resonance region [4] for the asymmetry -with no nuclear effects. Also shown as a solid curve is the prediction of Matsui, Sato and Lee [15] for the $\mathrm{N} \rightarrow \Delta$ transition in hydrogen at an incident beam energy of $4 \mathrm{GeV}$ at $Q^{2}=0.5 \mathrm{GeV}^{2}$.

The first uses a flash ADC (FADC) system to digitize the signals from the PID detectors and scintillators and passes them to an on board FPGA for processing. The logic in the FPGA would identify electrons, pions and associated pileup and count these in local memory which is read out to a VME bus once every helicity pulse. Alternatively a NIM based logic with a scaler counting algorithm can also be used to implement a counting scheme, at the expense of greater pileup and dead time due to the signal width needed for the scalers. More details may be found in Ref. [4,20].

As with any measurement of parity violation, careful control over systematic effects must be maintained. This is still true, even though the asymmetry measured in this experiment is relatively large $\left(A \approx 50 \times 10^{-6}\right)$ when compared with those of the completed HAPPEX II [29] $\left(A \approx 1 \times 10^{-6}\right)$ and proposed PREX $[30]\left(A \approx 0.5 \times 10^{-6}\right)$ experiments. The known sources of systematic uncertainties are listed in Tab. 2. The most significant of these uncertainties are discussed below.
Table 2. Sources of experimental systematic uncertainties and their estimated size. In comparing the ratios of the asymmetry from different targets, many of these uncertainties would cancel either partly or completely. For the target ratios, an overall systematic uncertainty of $\delta A / A \approx 0.010$ is estimated.

\begin{tabular}{lc}
\hline \hline Source & $\delta A / A$ \\
\hline Beam polarization & 0.012 \\
Kinematic determination of $Q^{2}$ & 0.009 \\
Electromagnetic radiative corrections & 0.005 \\
Beam asymmetries & 0.005 \\
Pion Contamination & 0.005 \\
Dead time corrections & 0.003 \\
Pair symmetric background & 0.002 \\
Target purity and density fluctuations & 0.002 \\
Pole tip background & 0.001 \\
\hline Total (in quadrature) & 0.018 \\
Total on target ratios & 0.010 \\
\hline \hline
\end{tabular}

The beam polarization would be measured with a combination of a Compton and a Møller polarimeter. An upgrade of the JLab Hall A Compton polarimeter is underway and needed for both the PREX and $6 \mathrm{GeV}$ PV-DIS experiments. Both the scattered photons and scattered electrons would be detected and the data may be analyzed independently or in coincidence. This upgrade would be able to achieve a combined statistical and systematic $1.2 \%$ measurement of the polarization, at the experiment's production beam current. This upgrade is expected to be complete in 2006. As a cross check, with lower beam current, the Hall A Møller polarimeter would have 1\% accuracy.

The experimental asymmetry, $A_{\text {Res }}$, is expected to be linearly proportional to $Q^{2}=4 E E^{\prime} \sin ^{2}(\theta / 2)$. In Hall A at JLab, the beam energy, $E$, can be determined to better than $\delta E<0.02 \%$ [27]. The scattered electron energy, $E^{\prime}$ as measured by either HRS spectrometer is known to $\delta E^{\prime}=0.1 \%$ and previous experiments have acheived a pointing resolution of $\delta \theta \leq 0.5 \mathrm{mr}$. When combined, the contribution of $\delta Q^{2}$ to the uncertainty of the asymmetry is expected to be $\delta A / A=0.9 \%$.

\section{Conclusion}

The Res-Parity experiment outlined in this talk would be the first measurement of parity violating electron scattering from hydrogen and deuterium targets covering the resonance region. The experiment has a large potential for discoveries in a kinematic region which is poorly explored with a weak probe. The experiment would test global quark-hadron duality in parity violation to $3 \%$ and local duality to better than $5 \%$. In addition to providing interesting data on the resonance region, Res-Parity would be able to constrain higher twist contributions to the $6 \mathrm{GeV}$ PV-DIS and $12 \mathrm{GeV}$ DIS-Parity experiments and would be important inputs to the radiative corrections which would be necessary for those experiments. 
Res-Parity would place constraints on the parity violating background in Møller scattering experiments both at SLAC [24] and at JLab [25,26]. The carbon and deuterium target data collected in this experiment would be used to study nuclear effects with the weak interaction. These data are especially interesting to the current generation of neutrino experiments which depend on nuclear targets for luminosity and scattering in and near the resonance region to search for neutrino flavor oscillations. Res-Parity is a relatively simple measurement of parity violation due to the large asymmetry, $A_{\mathrm{Res}} \approx 50 \times 10^{-6}$, and it is a natural experiment to be completed in association with the PV-DIS experiment (JLab E05-007)[20,21], using the same experimental equipment and technique.

This work was supported in part by the U.S. Department of Energy, Office of Nuclear Physics, under Contract Nos. DEAC05-84150 and W-31-109-ENG-38.

\section{References}

1. W. Melnitchouk, R. Ent, C. Keppel, Phys. Rept. 406, 127 (2005), hep-ph/0501217

2. K.A. Aniol et al. (HAPPEX), Phys. Rev. Lett. 96, 022003 (2006), nucl-ex/0506010

3. D.S. Armstrong et al. (G0), Phys. Rev. Lett. 95, 092001 (2005), nucl-ex/0506021

4. P. Bosted, J. Arrington, V. Dharmawardane, H. Mkrtchyan, X. Zheng et al., Parity violating electron scattering in the resonance region (Res-Parity) (2005), JLab P06-005

5. C.Y. Prescott et al., Phys. Lett. B77, 347 (1978)

6. C.Y. Prescott et al., Phys. Lett. B84, 524 (1979)

7. R.N. Cahn, F.J. Gilman, Phys. Rev. D17, 1313 (1978)

8. J.D. Bjorken, Phys. Rev. D18, 3239 (1978)

9. J.E. Amaro, M.B. Barbaro, J.A. Caballero, T.W. Donnelly, A. Molinari, Phys. Rept. 368, 317 (2002), nucl-th/0204001

10. M.J. Musolf et al., Phys. Rept. 239, 1 (1994)

11. M.B. Barbaro, J.A. Caballero, T.W. Donnelly, C. Maieron, Phys. Rev. C69, 035502 (2004), nucl-th/0311088

12. A. De Pace, M. Nardi, W.M. Alberico, T.W. Donnelly, A. Molinari, Nucl. Phys. A741, 249 (2004), nucl-th/0403023

13. D.R.T. Jones, S.T. Petcov, Phys. Lett. B91, 137 (1980)

14. L.M. Nath, K. Schilcher, M. Kretzschmar, Phys. Rev. D25, 2300 (1982)

15. K. Matsui, T. Sato, T.S.H. Lee, Phys. Rev. C72, 025204 (2005), nucl-th/0504051

16. T. Sato, T.S.H. Lee, Phys. Rev. C54, 2660 (1996), nucl-th/9606009

17. E.D. Bloom, F.J. Gilman, Phys. Rev. Lett. 25, 1140 (1970)

18. A. De Rujula, H. Georgi, H.D. Politzer, Ann. Phys. 103, 315 (1977)

19. D.M. Alde et al., Phys. Rev. Lett. 64, 2479 (1990)

20. X. Zheng, P. Reimer, R. Michaels et al., $e-{ }^{2} H$ parity violating deep inelastic scattering at CEBAF $6 \mathrm{GeV}$ (2004), JLab E05-007

21. X. Zheng, Parity Violation in Deep Inelastic Scattering at JLab $6 \mathrm{GeV}$, in PAVI '06: From Parity Violation to Hadronic Structure and more... (Part III) (Springer, Milos, Greece, 2006)
22. P. Reimer, K. Paschke, X. Zheng et al., Precision measurement of the parity-violating asymmetry in deep inelstic scattering off deuterium using baseline 12 GeV equipment in Hall $C$ (2006), JLab LOI-12-06-111

23. P. Souder, DIS-Parity at $12 \mathrm{GeV}$, in PAVI '06: From Parity Violation to Hadronic Structure and more... (Part III) (Springer, Milos, Greece, 2006)

24. P.L. Anthony et al. (SLAC E158), Phys. Rev. Lett. 95, 081601 (2005), hep-ex/0504049

25. D. Mack, Outlook of an Improved Measurement of Parity Violation in Moeller Sacttering at JLab (e2ePV), in PAVI '06: From Parity Violation to Hadronic Structure and more... (Part III) (Springer, Milos, Greece, 2006)

26. J. Arrington et al., eds., Pre-Conceptual Design Report $(p C D R)$ for The Science and Experimental Equipment for The 12 GeV Upgrade of CEBAF (Thomas Jefferson National Accelerator Facility, 2004)

27. J. Alcorn et al., Nucl. Instrum. Meth. A522, 294 (2004)

28. D.E. Wiser, Ph.D. thesis, University of Wisconsin (1977)

29. K.A. Aniol et al. (HAPPEX), Phys. Lett. B635, 275 (2006), nucl-ex/0506011

30. R. Michaels et al., A clean measurement of the neutron skin of ${ }^{208} \mathrm{~Pb}$ through parity violating electron scattering (2000), JLab E00-003 